\title{
Component Specification in the Cactus Framework: The Cactus Configuration Language
}

\author{
Gabrielle Allen \\ Center for Computation \& Technology \\ Department of Computer Science \\ Louisiana State University \\ Baton Rouge, Louisiana 70803 \\ Email: gallen@cct.lsu.edu
}

\author{
Tom Goodale \\ 7 Constantine Avenue, Heswall \\ Wirral CH60 5SU \\ United Kingdom \\ Email: goodale@gmail.com
}

\author{
Frank Löffler \\ Center for Computation \& Technology \\ Louisiana State University \\ Baton Rouge, Louisiana 70803 \\ Email: knarf@cct.lsu.edu
}

\author{
David Rideout \\ Perimeter Institute for Theoretical Physics \\ 31 Caroline St. N. \\ Waterloo, Ontario N2L 2Y5 \\ Canada \\ Email: drideout@perimeterinstitute.ca
}

\author{
Erik Schnetter \\ Center for Computation \& Technology \\ Department of Physics \& Astronomy \\ Louisiana State University \\ Baton Rouge, Louisiana 70803 \\ Email: schnetter@cct.lsu.edu
}

\author{
Eric L. Seidel \\ City College of New York \\ New York, New York 10031 \\ Center for Computation \& Technology \\ Louisiana State University \\ Baton Rouge, Louisiana 70803 \\ Email: eseide101@ccny.cuny.edu
}

\begin{abstract}
Component frameworks are complex systems that rely on many layers of abstraction to function properly. One essential requirement is a consistent means of describing each individual component and how it relates to both other components and the whole framework. As component frameworks are designed to be flexible by nature, the description method should be simultaneously powerful, lead to efficient code, and be easy to use, so that new users can quickly adapt their own code to work with the framework.

In this paper, we discuss the Cactus Configuration Language (CCL) which is used to describe components ("thorns") in the Cactus Framework. The CCL provides a description language for the variables, parameters, functions, scheduling and compilation of a component and includes concepts such as interface and implementation which allow thorns providing the same capabilities to be easily interchanged. We include several application examples which illustrate how community toolkits use the CCL and Cactus and identify needed additions to the language.
\end{abstract}

\section{INTRODUCTION}

Component frameworks provide a mechanism for efficiently developing and deploying scientific applications in highperformance computing environments. Such frameworks provide for efficient code reuse, community code development and abstraction of specialized capabilities such as adaptive mesh refinement or parallel linear solvers.

Component specification is obviously an important part of component frameworks with the specification providing the definition of the interfaces between components, including for example a description of the variables and functions both provided by and required by the different components. The choice of specification language impacts the scope of capabilities of components which can be implemented and exposed as well as the ease of use of components by both developers and users. If the component specification is too general it can hinder easy sharing of components, and if the specification is too narrow it will reduce the potential functionality of components and thus the application.

This paper describes the current specification of components in the Cactus Framework via the Cactus Configuration Language or CCL. Cactus is an open-source component framework designed for collaborative development of complex codes in high-performance computing environments. The largest user base for Cactus is in the field of numerical relativity where, for example, over 100 components are now shared among over fifteen different groups through the Einstein Toolkit [17] (Section IV-C). In other application areas, Cactus is used by researchers in fields including quantum gravity (Section IV-B), computational fluid dynamics, coastal modeling and computer science.

However, as simulation codes grow more complex, for example requiring multi-physics capabilities, there is now a need to extend or possibly re-architect the CCL to react to new features required by Cactus application developers. Further, as the number of Cactus components grow, an increasing problem is how to provide user tools for component assembly, application debugging, and verification and validation. This paper provides a review of the CCL focusing on how it describes the interactions between thorns and implications for the development of user tools.

In Section II we describe the architecture of the Cactus Framework that particularly relates to its handling and orchestration of components, including the Cactus Scheduler, memory allocation, data types provided by Cactus, and existing and planned tools for component management. In Section III we describe the Cactus Thorn configuration files using the Cactus Configuration Language, the methods of thorn interaction, and built-in testing options. In Section IV] we examine several different Cactus applications, the WaveToy Demo, a community 


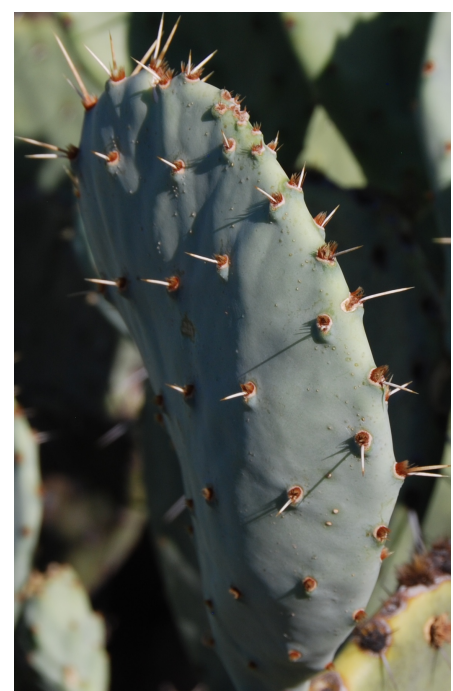

Fig. 1. Cactus components are called thorns and the integrating framework is called the flesh. The interface between thorns and the flesh is provided by a set of configuration files writing in the Cactus Configuration Language (CCL).

toolkit for quantum gravity, and the Einstein Toolkit, in respect to the dependence among components enforced by the CCL. In Section V] we describe some "missing" features of the CCL that will need to be addressed for future Cactus applications.

\section{CACTUS}

The Cactus Framework [16], [3] is an open source, modular, portable programming environment for HPC computing. It was designed and written specifically to enable scientists and engineers to develop and perform the large-scale simulations needed for modern scientific discoveries across a broad range of disciplines. Cactus is well suited for use in large, international research collaborations.

\section{A. Architecture}

Cactus is a component framework. Its components are called thorns whereas the framework itself is called the flesh (Figure 11). The flesh is the core of Cactus, it provides the APIs for thorns to communicate with each other, and performs a number of administrative tasks at build-time and run-time. Cactus depends on three configuration files and two optional files provided by each thorn to direct these tasks and provide inter-thorn APIs. These files are:

- interface.ccl Defines the thorn interface and inheritance along with variables and aliased functions.

- param.ccl Defines parameters which can be specified in a Cactus parameter file and are set at the start of a Cactus run.

- schedule.ccl Defines when and how scheduled functions provided by thorns should be invoked by the Cactus scheduler.

- configuration.ccl (optional) Defines build-time dependencies in terms of provided and required capabilities, e.g. interfaces to Cactus-external libraries.

\section{Cactus Thorn}

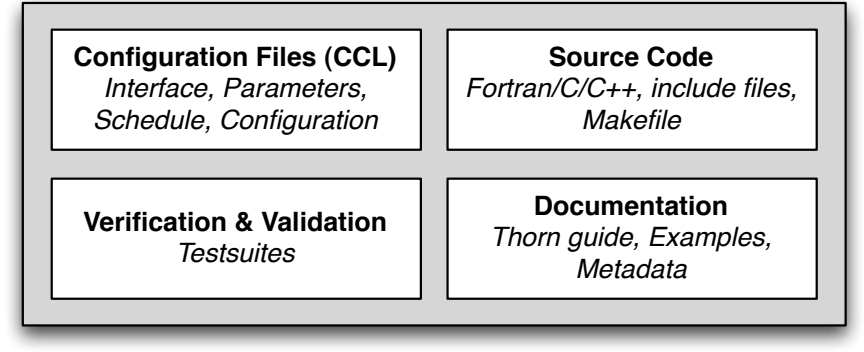

Fig. 2. Cactus thorns are comprised of source code, documentation, testsuites for regression testing, along with a set of configuration files written in the Cactus Configuration Language (CCL) which define the interface with other thorns and the Cactus flesh.

- test.ccl (optional) Defines how to test a thorn's correctness via regression tests.

The flesh is responsible for parsing the configuration files at build-time, generating source code to instantiate the different required thorn variables, parameters and functions, as well as checking required thorn dependencies.

At run-time the flesh parses a user provided parameter file which defines which thorns are required and provides keyvalue pairs of parameter assignments. ${ }^{1}$ The flesh then activates only the required thorns, sets the given parameters, using default values for parameters which are not specified in the parameter file, and creates the schedule of which functions provided by the activated thorns to run at which time.

The Cactus flesh provides the main iteration loop for simulations (although this can be overloaded by any thorn) but does not handle memory allocation for variables or parallelization; this is performed by a driver thorn. The flesh performs no computation of its own - this is all done by thorns. It simply orchestrates the computations defined by the thorns.

The thorns are the basic modules of Cactus. They are largely independent of each other and communicate via calls to the Flesh API. Thorns are collected into logical groupings called arrangements, This is not strictly required, but strongly recommended to aid with their organization. An important concept is that of an interface. Thorns do not define relationships with other specific thorns, nor do they communicate directly with other thorns. Instead they define relationships with an interface, which may be provided by multiple thorns. This distinction exists so that thorns providing the same interface may be independently swapped without affecting any other thorns. Interfaces in Cactus are fairly similar to abstract classes in Java or virtual base classes in $\mathrm{C}++$, with the important distinction that in Cactus the interface is not explicitly defined anywhere outside of the thorn.

This ability to choose among multiple thorns providing the same interface is important for introducing new capabilities in

\footnotetext{
${ }^{1}$ Note that this parameter file is different from the file param.ccl which is used to define which parameters exist, while the former is used to assign values to those parameters at run-time.
} 
Cactus with minimal changes to other thorns, so that different research groups can implement their own particular solver for some problem, yet still take advantage of the large amount of community thorns. For example, the original driver thorn for Cactus which handles domain decomposition and message passing is a unigrid driver called PUGH. More recently, a driver thorn which implements adaptive mesh refinement (AMR) was developed called Carpet [8], [7], [1]. Carpet makes it possible for simulations to run with multiple levels of mesh refinement, which can be used to achieve great accuracy compared to unigrid simulations. Both PUGH and Carpet provide the interface driver and application thorns can relatively straightforwardly migrate from unigrid to using the advanced AMR thorn.

Thorns providing the same interface may also be compiled together in the same executable, with the user choosing in the parameter file, at run-time, which implementation to use. This allows users to switch among various thorns without having to recompile Cactus.

Thorns include a doc directory which provides the documentation for the thorn in $\mathrm{ET}_{\mathrm{E}} \mathrm{X}$ format. This allows users to build one single reference guide to all thorns via a simple command.

\section{B. Scheduling}

The Cactus flesh provides a rule-based scheduler. Thorn functions can be specified to be called by the scheduler at different points in the simulation, in standard time bins. A scheduled routine can be requested to occur before/after other functions in the same timebin. It is also possible for thorns to define their own schedule groups, which may be thought of as a user-defined time bin. The specification of scheduled functions in thorns is described in Section III-A2 At run time, the flesh builds a schedule tree and provides an API that allows this schedule tree to be traversed such that the functions are called in their desired order. Cactus provides the argument lists for calling these scheduled functions, and provides information about which variables need storage allocated and when.

\section{Memory Allocation}

Memory allocation for Cactus variables is handled by the driver thorn, using information from the schedule and interface configuration files. Memory can be allocated for variables throughout the simulation, or allocated only during the execution of a function or schedule group. This provides a mechanism for reducing and tracking the memory footprint of a simulation. Incorrect memory allocation and the use of uninitialized variables can easily lead to bugs in codes which are hard to detect. Various Cactus thorns provide tools which help locate such errors, for example by initializing variables to have a value of $\mathrm{NaN}^{2}$ and then checking for these values during the simulation.

${ }^{2} \mathrm{~A}$ full explanation of NaN may be found online: http://en.wikipedia.org/ wiki/NaN

\section{Data Types}

Cactus defines its own data types for thorns. These data types include standard integer and real types, and a complex number data type. Supported Cactus data types include Byte, Int, Real, Complex, String, Keyword and Pointer, but the use of some of them is restricted (e.g. Keyword and String to parameters). An optional trailing number to the type can be used to set the size in bytes, where applicable. The motivation to provide Cactus data types comes from the fact that there is not a standard size for data types across all platforms. Providing Cactus-specific data types allows the framework to maintain an explicit variable size across all platforms, and provides maximum code portability. In addition it allows users to select the size of these standard types at build time across all thorns.

\section{E. Tools}

As a distributed software framework, Cactus can make use of some additional tools to assemble the code and manage the simulations. Oftentimes each arrangement of thorns resides in its own source control repository, as they are mostly independent of each other. This leads to a retrieval process that would quickly become unmanageable for end-users (for example the Einstein Toolkit is comprised of 135 thorns). To facilitate this process we use a thornlist written using the Component Retrieval Language [9], which allows the maintainers of a distributed framework to distribute a single file containing the URLs of the components and the desired directory structure. This file can then be processed by a program such as our own GetComponents script, and the entire retrieval process becomes automated.

In addition to the complex retrieval process, compiling Cactus and managing simulations can be a difficult task, especially for new users. There are a large number of options that may be required for a successful compilation, and these will vary across various architectures. To assist with this process a tool called the Simulation Factory [10], [15] was developed. Simulation Factory provides a central means of control for managing access to different resources, configuring and building the Cactus codebase, and also managing the simulations created using Cactus. Simulation Factory uses a database known as the Machine Database, which allows Simulation Factory to be resource agnostic, allowing it to run consistently across any pre-configured HPC resource.

\section{Cactus Configuration LAnguage}

The Cactus Configuration Language (CCL) was provided with the first Cactus 4.0 release in 1999. The language has evolved since then with the addition of function aliasing (Section III-A2) and the configuration CCL file (Section III-A), along with a small number of minor changes. The well designed initial capabilities and ensuing stability of the CCL is one feature of Cactus which has led to its success across different scientific fields and its ability to enable the growth of application communities. 


\begin{tabular}{|l|l|}
\hline Schedule Bin & Description \\
\hline \hline CCTK_STARTUP & $\begin{array}{l}\text { For routines which need to be run } \\
\text { before the grid hierarchy is set up, for } \\
\text { example, for function registration. }\end{array}$ \\
\hline CCTK_PARAMCHECK & $\begin{array}{l}\text { For routines that check parame- } \\
\text { ter combinations for potential errors. } \\
\text { Routines registered here only have ac- } \\
\text { cess to the grid size and the parame- } \\
\text { ters. }\end{array}$ \\
\hline CCTK_INITIAL & $\begin{array}{l}\text { For routines which generate initial } \\
\text { data. }\end{array}$ \\
\hline CCTK_PRESTEP & $\begin{array}{l}\text { Tasks performed before the main evo- } \\
\text { lution step. }\end{array}$ \\
\hline CCTK_EVOL & The evolution step. \\
\hline CCTK_POSTSTEP & $\begin{array}{l}\text { Tasks performed after the evolution } \\
\text { step. }\end{array}$ \\
\hline CCTK_ANALYSIS & $\begin{array}{l}\text { Routines which can analyze data at } \\
\text { each iteration. This time bin is special } \\
\text { in that ANALYSIS routines are only } \\
\text { called if output from the routine is } \\
\text { requested, e.g. in the parameter file }\end{array}$ \\
\hline
\end{tabular}

Fig. 3. Scheduled functions in Cactus can be assigned to run in standard time bins, the most important of which are described in this table.

In this section we outline the structure of the Cactus Configuration Language and provide syntax definitions for many of the elements of CCL. A complete specification and discussion of the language may be found in the Cactus User's Guide ${ }^{3}$.

\section{A. Thorn Configuration}

1) Groups: Cactus variables are placed in variable groups with homogeneous attributes, where the attributes describe properties such as the data type, variable group type, rank, dimensions, and number of time levels. Many Cactus functions operate on groups of variables, for example storage allocation, sychronization between processors, and output functions. For example, a vector field containing individual variables for fluid flow in different directions would typically include all the vector components in a single variable group. By default, all variable groups are private, however the public keyword can be used to change the access level for each subsequent variable group in the ccl file.

2) Functions: Cactus provides two types of functions, scheduled and aliased. Scheduled functions are declared in the schedule.ccl file and are defined to be called at certain stages in the Cactus simulation by prescribing a time bin, a specific time during a simulation, in which to run. Standard Cactus time bins are defined which are invoked in a well defined order, and a list of the most important Cactus standard time bins is provided in Figure 3

Additionally, thorn developers can define their own time bins or schedule groups. It is possible to specify the order in which two scheduled functions are called, as well as simple conditionals and loops. Memory allocation of Cactus variables can be restricted to only the time of execution of a certain

\footnotetext{
$\sqrt[3]{\text { http://cactuscode.org/documentation/UsersGuide.pdf }}$
}

function. Figure 4 shows a subset of the syntax which is used to define a scheduled function.

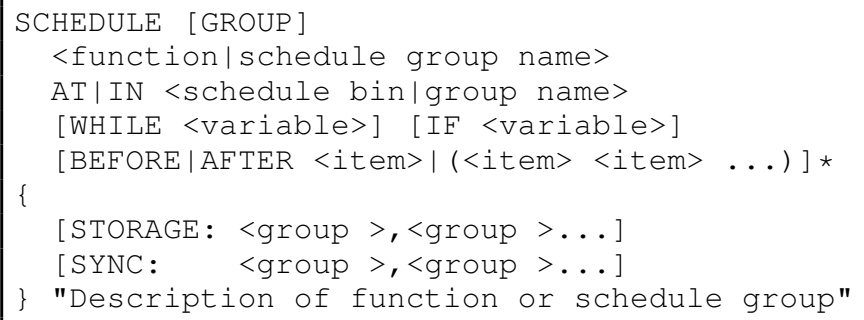

Fig. 4. Subset of the syntax for declaring scheduled functions or schedule groups of functions. A function can be scheduled at a certain time bin or in a schedule group. It can be called while or if a condition is fulfilled. Functions or schedule groups can be scheduled before or after other functions or schedule groups, within the same time bin or schedule group. Storage for Cactus variables might only be allocated for a certain function or schedule group, to save overall memory. Variables distributed over multiple processes can be automatically synchronized after a certain function or schedule group, if specified in the ccl file.

Aliased functions are functions that can be shared between thorns. They are declared in the interface.ccl file and may be called by a thorn at any point during the simulation. In order to call an aliased function it is not important to know the programming language used for its implementation. The Cactus API takes care of possibly necessary conversions.

3) Variables: Grid variables are Cactus variables that are passed between thorns by the flesh, and are declared in the interface.ccl file. They are generally collected into variable groups of the same data type. There are three types of variable groups: grid functions, arrays, and scalars. Grid functions (GFs), the most common variable group type, are arrays with a specific size set by the parameter file, which are distributed across processors. All GFs must have the same array size, typically defining the shape and size of the computational domain. Arrays are a more general form of GFs in that each array group may have a distinct size which can be given by Cactus parameters. Scalars are single variables of a given basic type, much like rank-zero arrays. Cactus variables can specify a number of timelevels, which means a certain number of copies of this variable for use in time-evolution schemes where data at a past time is needed to calculate the new data at a later time. Part of the syntax for declaring a variable group of variables is shown in Figure 5.

4) Parameters: Parameters are used to specify the runtime behavior of Cactus and are defined in the param. $\mathrm{ccl}$ file. They have a specific data type and scope, a range of allowed values, and a default value. Once parameters have been set, they cannot be modified unless specifically declared to be steerable, in which case they may be dynamically changed throughout the simulation. The allowed datatypes for parameters are Int, Real, Keyword, Boolean, and String. Thorns can use and extend parameters of other thorns. The syntax for declaring Cactus parameters is shown in Figure 6

5) Include Files: Header files can be shared between thorns if specified in the interface.ccl file. It is not only 


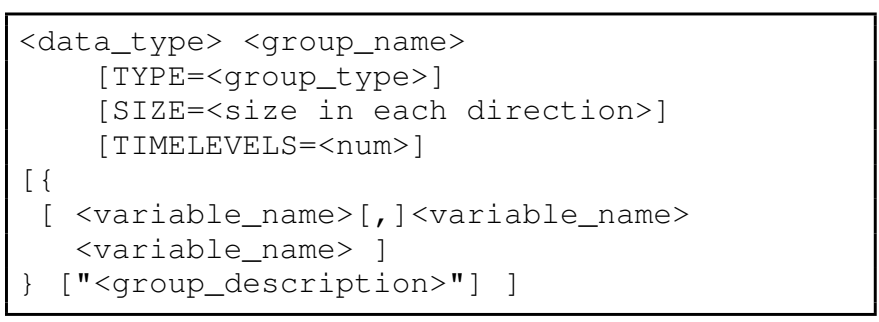

Fig. 5. Part of the syntax for declaring Cactus variables. Cactus variables have to be one of the data types Cactus defines and are part of a variable group. They can have different Cactus variable types, sizes, and number of time levels. Each variable group needs to have a human-readable description.

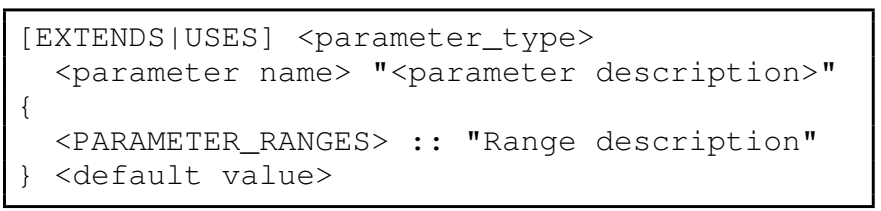

Fig. 6. Syntax for declaring Cactus parameters. Thorns might use or extend parameters of other thorns, and define their own. A parameter needs to have a data type. A human-readable description needs to be given, as well as an allowed range with a description for the range and a default value within that range.

possible to share a single include file, but also to concatenate multiple include files (also from multiple thorns), and use them like a single include file. During the build process, Cactus copies all of the source files located in each thorn's include directory to a central location from which they may be accessed by any other thorn using one of two methods shown in Figure 77. USES INCLUDE requests an include file from another thorn, and INCLUDE adds the code in $<f i l e \_t o \_i n c l u d e>$ to $<f i l e \_n a m e>$.

USES INCLUDE: <file name>

INCLUDE[S] : <file_to_include> IN <file_name>

Fig. 7. Syntax for using include files in Cactus. Thorns might provide a specific header file to another thorn (the first example), or might provide one part of a concatenation of multiple header files, possibly from multiple thorns (the latter example).

\section{B. Thorn Interaction}

1) Scope: Cactus provides different levels of access for variables and parameters. Variables can be defined as public or private. Public variables can be inherited by a thorn when that thorn inherits an interface. Thorn inheritance will be described in greater detail below. Private variables can only be seen by the thorn which defines them.

Similarly, parameters may be defined as restricted or private. Restricted parameters are available to thorns which request access. Private parameters, like variables, are only visible to the thorn which defines them. The access levels here only specify if those parameters are directly accessible in the source code; it is possible to access information about any parameter through Cactus API functions regardless of the parameter scope defined in the param. $\mathrm{ccl}$ file.

2) Inheritance: Cactus provides an inheritance mechanism similar to Java's abstract classes. It allows thorns to gain access to variables provided elsewhere by inheriting from the interface. A key point here is that the thorns are not inheriting from other specific thorns; any number of thorns may declare themselves as implementing an interface. These thorns may all be compiled together, allowing the user to decide at run-time which thorn should be used. The interface is only specified by the thorns implementing it. This means that thorns declaring the same interface-name need to have an identical interface, which is checked by Cactus.

Cactus also provides capabilities which may be declared in the configuration. $\mathrm{CCl}$ file. Capabilities differ slightly from interfaces in that while any number of thorns providing the same interface may be compiled together, only one thorn providing a capability may be compiled into a specific configuration. In this sense, while interfaces define run-time dependencies, capabilities define build-time dependencies. This can be useful for providing external libraries or functions which are too complex for aliasing. Also, capabilities play a role in configuring thorns and external libraries since they interact with the build system of Cactus.

Many design decisions are based on the distinction between interfaces and capabilities. For example, the concept of capabilities is important for application performance - knowing an inter-thorn relationship at build time allows optimizations to be included that are not possible at run time.

The syntax for declaring and requiring a capability is shown in Figure 8.

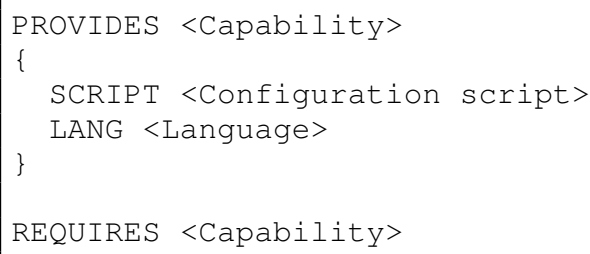

Fig. 8. Part of the syntax for declaring and requiring capabilities in Cactus. Capabilities can be required and provided by thorns. If a thorn provides a capability it interacts with the makesystem through the output of a script which needs to be specified in the ccl file, as well as it's programming language to be able to call it correctly.

The interface.ccl file also provides a low-level include mechanism, described in Section III-A5, similar to that found in $\mathrm{C} / \mathrm{C}++$. Thorns may request access to any include file within the Cactus source tree without specifying which thorn or interface should provide it. This is used primarily for optimization reasons as the compiler can then replace inline functions, and in some cases for providing access to external libraries such as HDF5.

\section{Testing}

It is strongly recommended, although not required, that thorns come with one or more test suites. These consist of 
sample parameter files and the expected output for those parameters. These files should be located within the test directory in the thorn, so that the test suites may be run using gmake <configuration>-testsuite. These test suites serve the dual purposes of regression and portability testing.

\section{EXAMPLES}

In this section we show some examples of the dependencies among Cactus thorns which are generated by the CCL files for different applications: a simple example application for the scalar wave equation with a minimal set of thorns; a small community toolkit for quantum gravity; and a large community toolkit for numerical relativity. The interest on thorn dependencies arises for two core reasons:

1) Cactus is particularly targeted at enabling communities to generate shared toolkits for solving a variety of problems in a particular field. The standard computational toolkit which is distributed with Cactus is further used by many different applications. Thorn dependencies and interfaces thus need to be carefully thought out and periodically revisited to make sure that the plug-andplay aim of Cactus, where different thorns can provide the same functionality, is achieved with interfaces which are as simple, flexible and general as possible. This design usually involves a delicate balance, taking into account the speed of implementation, complexity of the interface etc.

2) Long time Cactus users work with standard thorn lists which are built up from experience and shared with collaborators. These thorn lists are amended as new thorns become available or are no longer used, and can contain several hundred thorns. For new users in particular, there is an increasing issue with providing a procedure for users to select the appropriate set of thorns for their application, and to understand the capabilities of different thorns. One big simplification which could be made would be to reduce the number of thorns in thorn lists by removing thorns which depend on others and could be automatically added. Ideally, a tool would be built which would allow a user to start from an abstract description of their problem and automatically select appropriate thorns, for example Evolving Gaussian initial data using the 3-D scalar wave equation and outputting 3D data, or Evolving two black holes using Einstein's equations and calculating gravitational waveforms. The question is then whether there is currently enough information in the CCL files to achieve this, or how additional information could be provided.

In this section, we use the dependencies among the sets of thorns described in the CCL files for these three example applications to view the complete set of thorn dependencies and to investigate how the thorn set could potentially be generated from an initial minimal set of thorns. The dependencies used for the figures are taken from a file generated during the
Cactus build process which contains a complete database of the contents of the different thorn configuration files.

A Perl script is used to parse this database and generate a file in dot format, which can then be processed by a program like graphviz [12] and turned into a directed graph like that in Figure 9. This graph shows five different types of dependencies. Inheritance is denoted by a regular arrow, dependencies due to a required function are denoted by an arrow with a square head, direct thorn dependencies are denoted by a dotted arrow, shared variable dependencies are denoted by an arrow with a circular head, and dependencies due to a required capability are denoted by an arrow with a diamond head. There are also shaded and unshaded thorns, the distinction being that the shaded thorns have no other thorns depending on them.

This Perl script does not show the dependencies generated by a single thorn, so we also use a set of two Python scripts, the first of which parses the actual CCL files and generates an XML file containing all of the dependencies. This file can then be queried by the second script, which will search for a single thorn and find all thorns upon which the query depends. It will also output a graph in dot format, as seen in Figure 10 The second script will also allow users to choose between alternate implementations of the same interface (e.g. PUGH or carpet). The motivation here is that this script should allow the user to generate a complete thornlist that could then be used to build a simulation.

\section{A. Simple Example: Scalar Waves}

The set of Cactus thorns to solve the 3-D scalar wave equation (WaveToy Demo) was developed as a pedagogical example for understanding Cactus, and as a simple and well understood test case for new developments. These thorns solve the hyperbolic wave equation in 3D Cartesian coordinates with different boundary conditions for a chosen set of initial data and include different output formats and a web interface. This example is described on the Cactus web pages [16], which also provide a thorn list with information about the 22 thorns that are used. The example application includes two initial data thorns which specify the initial scalar field and sources (idscalarwavec and wavebinarysource), a scalar field evolver (wavetoyc) along with additional thorns from the standard Cactus Computational Toolkit. The example uses the unigrid driver pugh with associated thorns pughslab for hyperslabbing and pughreduce which provides a set of standard reduction operations that can calculate for example the maximum value or L2 norm over the grid for any grid variable.

A complete set of dependencies between these thorns as specified in the CCL files is shown in Figure 9 In this diagram we can see for example the central nature of the ioutil thorn which provides functionality that can be used by thorns implementing different $\mathrm{I} / \mathrm{O}$ methods, for example providing a parameter which sets when data for all I/O methods should be output and the directory in which to write data. 


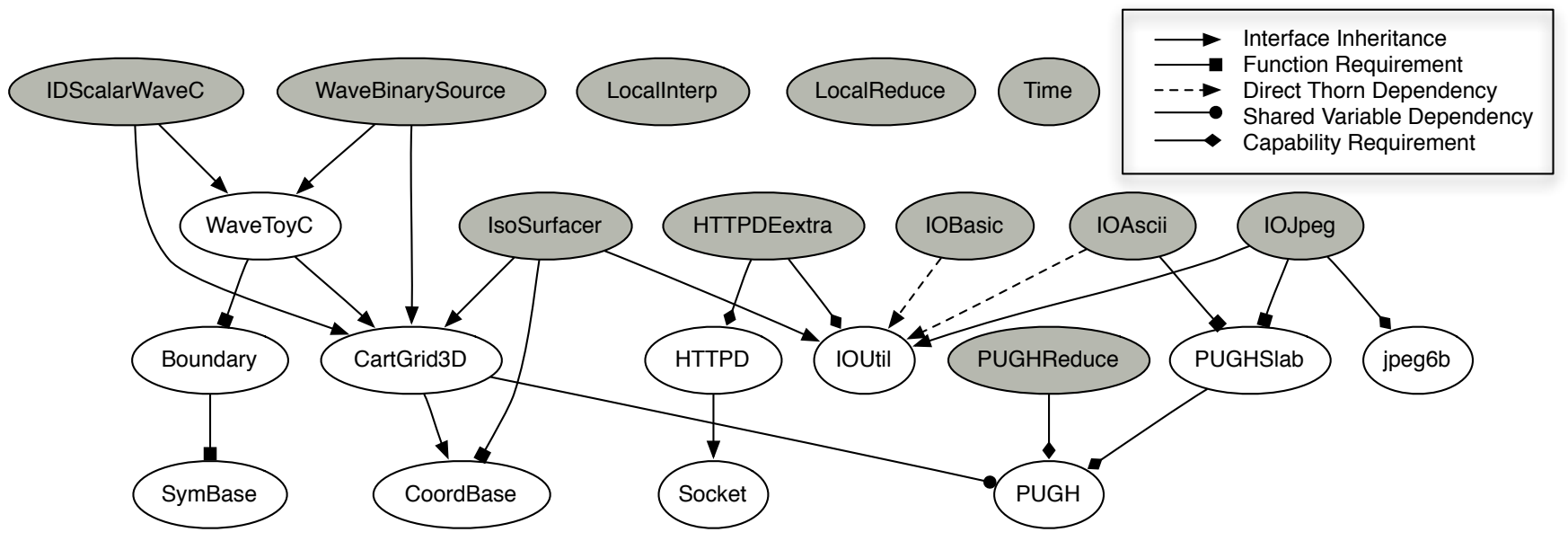

Fig. 9. Dependency graph for complete set of thorns in the simple example application WaveToy Demo. The shaded items indicate that the thorns are 'leaves' and have no thorns depending on them.

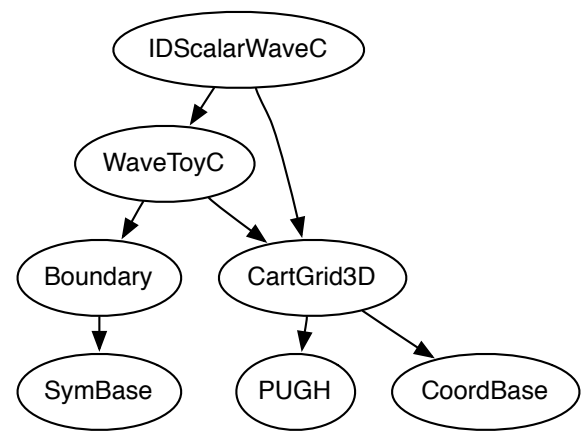

Fig. 10. Dependency graph for the WaveToy Demo thornlist. This graph is generated using dependencies of thorn IDScalarWaveC which defines initial data for the fields evolved by the scalar wave equation.

The dependency diagram also shows that any method to automatically generate this set of thorns using dependency information would need 11 thorns specified as a starting point, these are the shaded thorns in the diagram. For example, if we simply started from the thorn that specifies the initial scalar field (idscalarwavec) as shown in Figure 10, which could be the obvious starting point for a user who knows they want to evolve a particular scalar field then working only with dependencies would result in a set of thorns without using any coordinate time (time), any I/O, or the possibility to include scalar source terms.

Adding additional metadata to thorns is one mechanism to supplement the current CCL information to enable the generation of thorn lists for a particular application. For example, explicitly tagging thorns as providing I/O methods would allow these thorns to be automatically added or to be selected by a user. In other cases, these diagrams show that additional interfaces or dependencies may need to be added. In Figure 10 attention needs to be given to the compile time dependencies that would include thorns time (which should in fact be inherited by the evolution thorn) and PUGHReduce and localreduce.

\section{B. Small Community Code: The CausalSets Toolkit}

The CausalSets Toolkit is an example of a small community codebase, which implements a wide variety of computations in discrete quantum gravity, in particular with regard to Causal Set Theory [13]. The toolkit is based upon two major components. One is a MonteCarlo arrangement, which provides a generic API for providing parallel random numbers, i.e. pseudo random numbers which are independent on all processes. A second is a CausetBase API, provided by the BinaryCauset thorn, which abstracts the mathematical notion of a causal set (a locally finite partially ordered set [13]), providing myriad routines for working with such objects.

One of the challenges in supporting computations in Causal Set Theory is that there is not a single sort of computation, such as finding approximate solutions to PDEs by finite difference or spectral methods, which one would like to perform. Instead a physicist will ask many different sorts of questions about the behavior of discrete partial orders. A given computation will share aspects with others, but the overall structure may differ considerably. Furthermore the community is in general not terribly experienced with large scale computation, and thus benefits from software which insulates the physicist from many complications of parallel computing. The component based approach provided by the Cactus Framework is well suited to address both of these challenges, by allowing the physicist to mix and match individual components to build up the particular computation desired, working with familiar abstract mathematical concepts, rather than having to work directly with source code. Additionally the components are designed to run readily on large scale hybrid architectures, without the user needing detailed knowledge of how the computation is implemented.

The dependency diagram for a collection of thorns which implements a sample computation is shown in Figure 11. This 
is a computation of spatial homology of a sprinkled causal set, as described in [4]. Here the BinaryCauset thorn implements the core CausetBase API, which provides the causal set along with a high level abstract interface to it. The MonteCarlo thorn provides parallel random numbers to CFlatSprinkle, which generates a random causal set, and RandomAntichain, which selects a random antichain within the causal set provided by CFlatSprinkle. The MonteCarlo arrangement gets the actual pseudorandom numbers from thorn RNGs, and also provides a thorn Distributions to provide samples from a variety of distributions, such as Poisson and Gaussian. AntichainEvol provides a sequence of 'thickened antichains', which are then read by the Nerve thorn, which computes a nerve simplicial complex from each thickened antichain. The homology groups of these simplicial complexes are then computed by a separate standalone homology package chomp [2]. The whole computation relies on PUGH as a standard Cactus driver, and uses Cactus' IOUtil to provide metadata for IO routines.

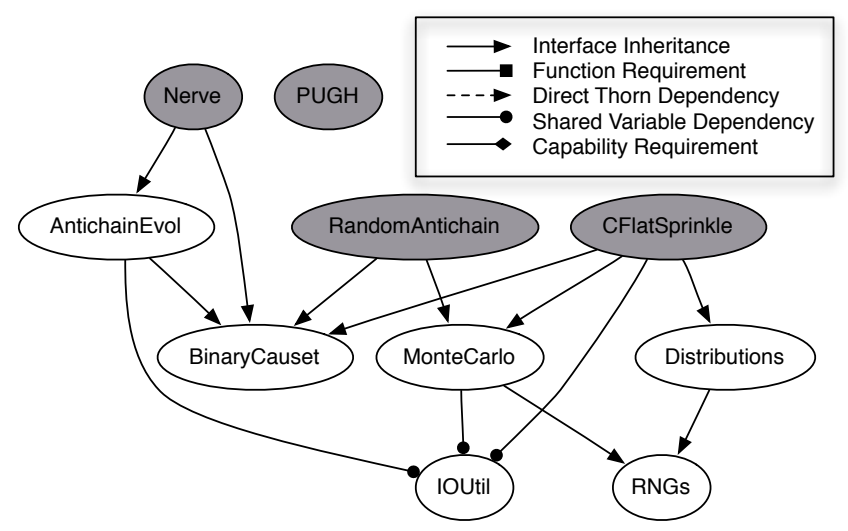

Fig. 11. Dependency graph for a sample computation in Causal Set Quantum Gravity. The computation is described in detail in [4].

\section{Large Community Code: The Einstein Toolkit}

The Einstein Toolkit [17] is an open, community developed software infrastructure for relativistic astrophysics. The Einstein Toolkit is a collection of software components and tools for simulating and analyzing general relativistic astrophysical systems that builds on numerous software efforts in the numerical relativity community. The Cactus Framework is used as the underlying computational infrastructure providing large-scale parallelization, general computational components, and a model for collaborative, portable code development. The toolkit includes modules to build complete codes for simulating black hole spacetimes as well as systems governed by relativistic hydrodynamics. Current development in the consortium is targeted at providing additional infrastructure for general relativistic magnetohydrodynamics.

The Einstein Toolkit uses a distributed software model and its different modules are developed, distributed, and supported either by the core team of Einstein Toolkit Maintainers, or by individual groups. When modules are provided by external groups, the Einstein Toolkit Maintainers provide quality control for modules for inclusion in the toolkit and help coordinate support.

With such a large set of components and a distributed team of developers, implementing appropriate standards are crucial to maintain coherence across the code base, and to enable future development. This is achieved in some part by defining base thorns that act to define application specific standards, providing default variables, parameters, functions and schedule bins that are common across an application. For example, in the Einstein Toolkit application specific base thorns include ADMBase (for the vacuum spacetimes), HydroBase (for matter spacetimes) and EOSBase (for equations of state) [6].

Figure 12 shows the complete dependency graph for the Einstein Toolkit, which is so extensive that it isn't possible to examine in detail in print ${ }^{4}$; however, we include the graph here to illustrate its complexity. Of the 135 thorns, 9 have no dependency on other thorns, and 78 thorns (including these independent thorns) are needed as the starting point to generate the whole toolkit using CCL dependency information. The clusters of dependencies for ADMBase, HydroBase and EOSBase are apparent in the diagram.

The Einstein Toolkit dependency diagram also shows a number of direct thorn dependencies, indicated by the black dotted lines. This means that thorns depend not on an interface but on a specific thorn. In some cases this is due to missing general interfaces such as appropriate aliased functions which either need to be carefully designed or perhaps have simply not been added where they should have been. A large number of these direct dependencies are associated with the Carpet adaptive mesh refinement set of thorns where the nature of the driver thorn typically enforces a direct dependency for example for associated I/O or reduction operations. The need to support direct dependency on thorns was one reason why the configuration. CCl file was introduced as an extension to the original CCL.

Figure 13 shows an example of the direct dependencies for an initial data thorn in the Einstein Toolkit. The thorn IDAnalyticBH provides initial data for several different black hole spacetimes with analytic solutions. Starting from this thorn, only seven other thorns are picked up directly with dependency information. Given that most production runs for numerical relativity simulations include of order 100 thorns, it is clear that automatically generating appropriate thorn lists will require additional metadata and physics insight.

\section{Future WORK}

The original Cactus Configuration Language was released as part of the Cactus 4.0b distribution in 1999 and has since that time been extended in different ways as new features were required. Despite serving the Cactus user community well since this time, it is clearly time to reexamine the requirements for the CCL in the light of current and future needs and to

\footnotetext{
${ }^{4}$ Note that if viewing this paper as a PDF document it is possible to zoom in to see features in detail.
} 


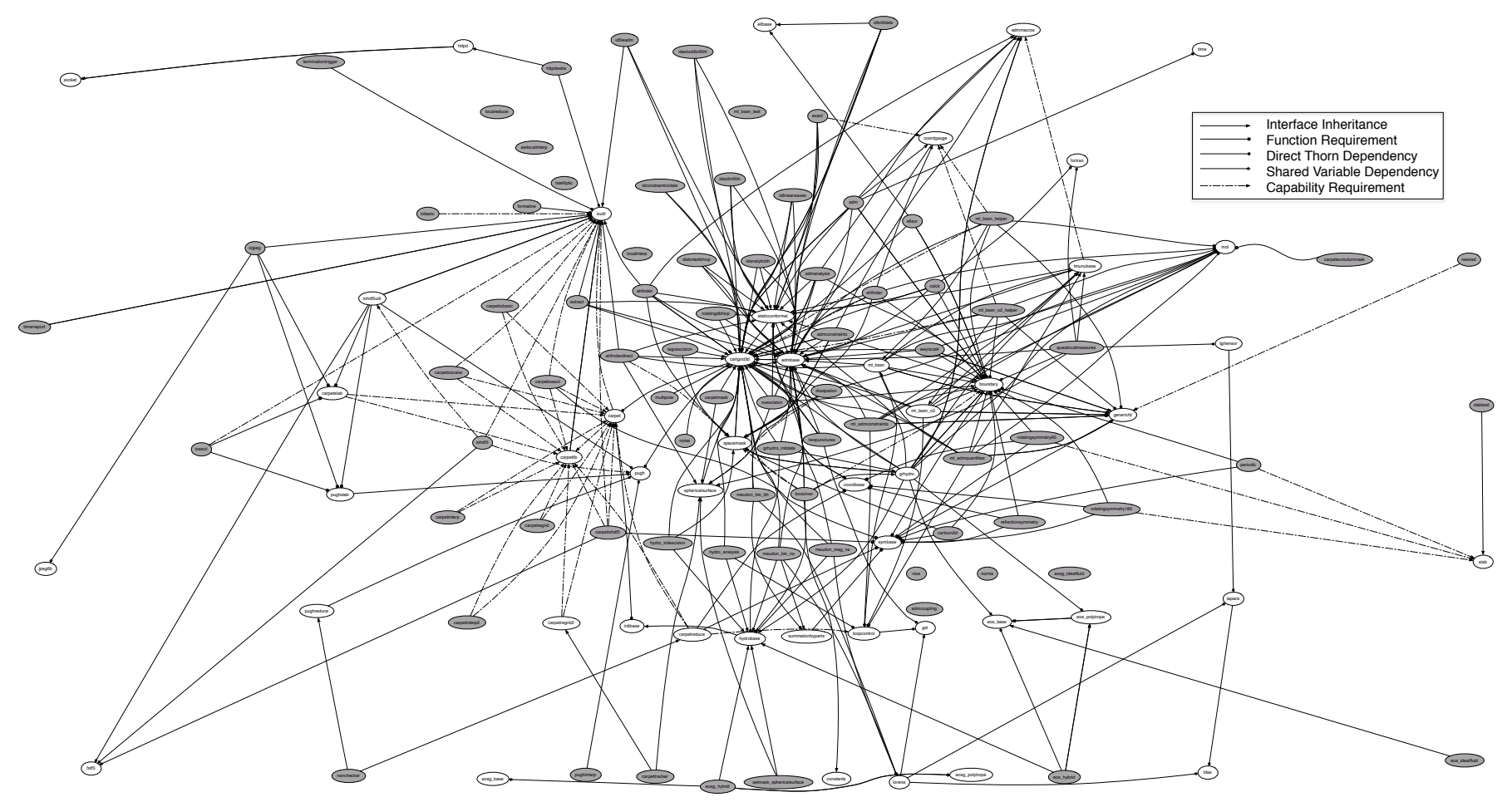

Fig. 12. Complete dependency graph for the 135 thorns of the EinsteinToolkit (http://www.einsteintoolkit.org)

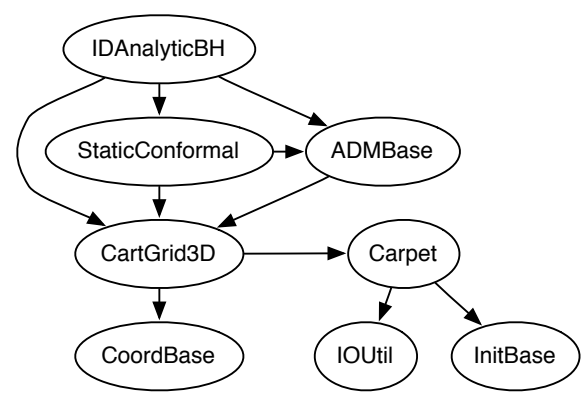

Fig. 13. Dependency graph for the Einstein Toolkit starting from the IDAnalyticBH thorn. For this graph the thorn Carpet was chosen to provide the driver interface, however PUGH could have been used instead.

take into account new technologies and possibilities. In this section we describe new features required in the CCL and their motivation.

Cactus (and the set of thorns in the Cactus Computational Toolkit) currently best supports finite difference, finite volume, or finite element methods implemented on structured grids. Extensions to the CCL are required to support meshless methods (e.g. particle methods such as smoothed particle hydrodynamics or particle-in-cell, used for example in many astrophysics codes) and unstructured meshes where additional connectivity information is required to specify how grid points are connected (e.g. unstructured grids are important for example in coastal modeling to resolve the fine details of the coastline). Implementing both these features in Cactus requires developing appropriate parallel driver and associated infrastructure thorns in addition to changes to the CCL.

Cactus currently operates with a single computational grid so that all physical models need to run on a single domain. Comprehensive multiphysics support is needed where different physical models can be configured and run on different domains, for example for coupling together wind and current models in coastal science, or modeling different physical components of a relativistic star.

Constants (e.g. $\pi$ or the solar mass) are commonly used in scientific codes. Currently in Cactus constants are handled via include files, for example the Einstein Toolkit contains a thorn which provides commonly used astrophysical constants in an include file. These constants are then only available in source code and not in CCL files. A preferable approach would be to define such constants directly as part of the CCL specification.

Similar to constants, the CCL needs to support enumerations and user-defined structures, so that e.g. a hydrodynamical state vector consisting of density, velocity, and temperature can be handled as a combined entity instead of as a set of five separate variables. This should include the ability to handle vectors and tensors in a natural manner, a feature that is missing in many computer languages, but which is nevertheless important in physics simulations. Tensor support would need to include support for symmetries (so that e.g. only 6 out of 9 components of the stress tensor are stored). In implementing this, it is important that the abstract specification of data types is decoupled from the decision of how to lay them 
out in memory, which needs to be left to the driver to ensure the highest possible performance on modern architectures that may offer vectorization and deep cache hierarchies.

While Cactus, through the CCL, contains information on how thorns fit together computationally the CCL does not contain information on the scientific content of the thorns. This issue needs some attention as the number of thorns in particular domains grows and models become more complex. Options to handle this could include extending the CCL, or adding descriptive metadata separate to the CCL, or by investigating whether enough information can be provided from the CCL and base thorns for a particular application. Such additional information is important, for example, to be able to automatically construct appropriate thornlists for a particular physical model.

A further issue related to the growth in both the number of thorns and the complexity of applications is constructing and editing CCL files. CCL files for some thorns are now very long and complex and difficult to read and comprehend. This issue could be addressed by restructuring the CCL itself or by providing intuitive and flexible higher level tools for interpreting, checking and editing files.

A final consideration is the syntax for the CCL. Changing the CCL syntax could improve the ease with which the files could be constructed and edited, and importantly provide more options for standard tools which could be used to construct, investigate, debug and edit the CCL files. As an example, using a standardized syntax for CCL would allow users to take advantage of the extensive features of the Eclipse Platform [5]. Eclipse is an advanced Integrated Development Environment $(\mathrm{IDE})^{5}$ that includes features such as customizable syntax highlighting, auto-completion of code, and dynamic syntax checking for languages it recognizes. One option for revising the CCL syntax would be to use an existing data markup language that incorporates metadata such as the Resource Description Framework (RDF) [14]. RDF is a widely used standard for describing data in internet tools. It uses URIs to describe the relationship between two objects as well as the two ends of the link, which is commonly known as a triple. This would be a natural method for describing the dependencies between thorns, however RDF is generally used as an extension of XML, which is not easily readable by humans. As the CCL files must be generated by hand, it would be preferable to use an alternate format that focuses on readability. One such example is YAML (YAML Ain't Markup Language) [11], a data serialization language with a strong emphasis on human readability. YAML represents data as a series of sequences and mappings, both of which can be nested within others. While YAML does not inherently support metadata, it would be quite simple to add metadata to the thorns by adding extra mappings to the CCL files.

\section{CONCLUSION}

We have presented an overview of the Cactus Configuration Language (CCL) that describes Cactus thorns and have shown

5 http://en.wikipedia.org/wiki/Integrated_development_environment how the CCL is used in three different applications. The dependency information included in the CCL specification can be used to identify potential issues in designing complex codebases, and to build high-level tools to better assist users in constructing codes for particular applications.

New features needed in the CCL specification have been identified, including support for more numerical methods, multiple physical models, user-defined structures, scientific metadata and to address the growing complexity of interfaces.

\section{ACKNOWLEDGMENT}

The development of Cactus and the CCL has been a long term and ongoing effort with many contributors and funders. In particular we acknowledge the contributions of Gerd Lanfermann, Joan Massó, Thomas Radke, and John Shalf, and funding from the National Science Foundation, MaxPlanck-Gesellschaft, and Louisiana State University. We also acknowledge colleagues in the Einstein Toolkit Consortium whose thorns provide the motivation and core use case for this work.

Work on thorn dependencies was funded by NSF \#0904015 (CIGR) and NSF \#0721915 (Alpaca). Eric Seidel acknowledges support from the NSF REU program (\#1005165) and thanks the Center for Computation \& Technology for hosting his undergraduate internship.

\section{REFERENCES}

[1] Mesh Refinement with Carpet, URL http://www.carpetcode.org/

[2] Pawel Pilarczyk et. al., Chomp, http://chomp.rutgers.edu.

[3] T. Goodale, G. Allen, G. Lanfermann, J. Massó, T. Radke, E. Seidel, and J. Shalf, The Cactus framework and toolkit: Design and applications, Vector and Parallel Processing - VECPAR'2002, 5th International Conference, Lecture Notes in Computer Science (Berlin), Springer, 2003.

[4] Seth Major, David Rideout, and Sumati Surya, Stable homology as an indicator manifoldlikeness in causal set theory, Class.Quant.Grav. 26 (2009), no. 175008, eprint 0902.0434 [gr-qc].

[5] Eclipse: An open development platform, URL http://www.eclipse.org/

[6] Erik Schnetter, Multi-physics coupling of einstein and hydrodynamics evolution: a case study of the einstein toolkit, CBHPC '08: Proceedings of the 2008 compFrame/HPC-GECO workshop on Component based high performance (New York, NY, USA), ACM, 2008, pp. 1-9.

[7] Erik Schnetter, Peter Diener, Nils Dorband, and Manuel Tiglio, A multiblock infrastructure for three-dimensional time-dependent numerical relativity, Class. Quantum Grav. 23 (2006), S553-S578, eprint grqc/0602104, URL http://stacks.iop.org/CQG/23/S553

[8] Erik Schnetter, Scott H. Hawley, and Ian Hawke, Evolutions in 3D numerical relativity using fixed mesh refinement, Class. Quantum Grav. 21 (2004), no. 6, 1465-1488, eprint gr-qc/0310042.

[9] Eric L. Seidel, Gabrielle Allen, Steven Brandt, Frank Löffler, and Erik Schnetter, Simplifying complex software assembly: the component retrieval language and implementation, TG '10: Proceedings of the 2010 TeraGrid Conference (New York, NY, USA), ACM, 2010, pp. 1-8.

[10] SimFactory: Herding Numerical Simulations, URL http://www.cct.lsu. edu/ eschnett/SimFactory/

[11] The Official YAML Web Site, URL http://yaml.org

[12] Graphviz Graph Visualization Software, URL http://www.graphviz.org

[13] Rafael D. Sorkin, Causal sets: Discrete gravity, Lectures on Quantum Gravity, Proceedings of the Valdivia Summer School, Valdivia, Chile, January 2002 (A. Gomberoff and D. Marolf, eds.), Plenum, 2005, eprint gr-qc/0309009.

[14] RDF Semantic Web Standards, URL http://www.w3.org/RDF

[15] Michael Thomas and Erik Schnetter, Simulation factory: Taming application configuration and workflow on high-end resources, CBHPC ' 10 (New York, NY, USA), ACM, accepted.

[16] Cactus Computational Toolkit, URL http://www.cactuscode.org

[17] The Einstein Toolkit, URL http://www.einsteintoolkit.org 